
\title{
Research Suare $=$ \\ Live imaging and conditional disruption of native vertebrate planar cell polarity
}

\section{Maria Jussila}

The Hospital for Sick Children

\section{Curtis Boswell}

Yale University School of Medicine

Brian Ciruna ( $\nabla$ ciruna@sickkids.ca )

The Hospital for Sick Children

\section{Article}

Keywords:

Posted Date: August 18th, 2021

DOI: https://doi.org/10.21203/rs.3.rs-764931/v1

License: (1) This work is licensed under a Creative Commons Attribution 4.0 International License. Read Full License

Version of Record: A version of this preprint was published at Nature Communications on September 23rd, 2022. See the published version at https://doi.org/10.1038/s41467-022-33322-9. 
1 Live imaging and conditional disruption of native vertebrate planar cell polarity

3 Maria Jussila ${ }^{1}$, Curtis W. Boswell ${ }^{1,2,3}$ and Brian Ciruna ${ }^{1,2, *}$

4

5 1. Program in Developmental \& Stem Cell Biology, The Hospital for Sick Children, 686 Bay

6 Street, Toronto, Ontario, M5G 0A4, Canada.

7 2. Department of Molecular Genetics, The University of Toronto, Toronto, Ontario, M5S 1A8, 8 Canada.

9 3. Current address: Department of Genetics, Yale University School of Medicine, New Haven, 10 CT 06510, USA.

$11 *$ Corresponding author. Email: ciruna@sickkids.ca

12 


\section{Abstract}

21 Tissue-wide coordination of polarized cytoskeletal organization and cell behaviour, critical for

22 organ morphogenesis and tumour progression, is controlled by asymmetric membrane

23 localization of non-canonical Wnt/planar cell polarity (PCP) signalling components.

24 Understanding the dynamic regulation of PCP thus requires visualization of these core proteins.

25 In vertebrates, immunohistochemical studies have provided snapshots of PCP within tissues

26 while exogenous, fluorescently-tagged reporters have been introduced for live imaging of cell

27 polarity. However, the functionality and spatiotemporal relevance of static and exogenous PCP

28 readouts remain uncertain. Here we fluorescently tag an endogenous core PCP component,

29 Vang12, in zebrafish. We report on the authentic regulation of vertebrate PCP, through live

30 imaging of this native sfGFP-Vangl2 protein during embryogenesis. We couple sfGFP-Vang12

31 with conditional zGrad GFP-nanobody degradation methodologies for tissue-specific

32 interrogation of PCP function. Together, our studies provide crucial insights into the

33 establishment and maintenance of vertebrate PCP and create a powerful experimental paradigm

34 for future investigations.

\section{Introduction}

36 The non-canonical Wnt/planar cell polarity (PCP) signalling pathway controls polarized, uniform

37 orientation of cells within a plane of a tissue. PCP pathway has been shown to regulate a number

38 of developmental and homeostatic processes by directing collective asymmetric modification of

39 the cell cytoskeleton, leading groups of cells to either move or divide in a shared direction, or

40 position their organelles such as the basal body asymmetrically ${ }^{1-5}$. Molecular genetic studies in

41 Drosophila have identified essential roles for asymmetric membrane localization of opposing

42 PCP signalling proteins in both the establishment and intercellular propagation of polarity ${ }^{6}$. 
43 Despite identification of a multitude of PCP-regulated morphogenetic processes, characterization

44 of vertebrate PCP at the cellular and molecular level is still lacking. This is challenging, because

45 detailed observation requires live imaging capabilities and the ability to differentiate asymmetric

46 protein localization across membranes of adjacent cells. In vertebrates, immunohistochemical

47 studies have provided snapshots of PCP within tissues ${ }^{7,8}$ while exogenous, fluorescently-tagged

48 reporter proteins have been introduced for mosaic imaging of PCP. Although great complexities

49 in the dynamic regulation of PCP have been reported across tissues and polarized cell

50 behaviours, ${ }^{9-12}$ the functionality and spatiotemporal accuracy of these static and exogenous

51 readouts remain uncertain. The biological relevance of studies utilizing exogenous,

52 fluorescently-tagged PCP molecules remains to be determined, as overexpression of PCP

53 proteins can disrupt cell polarity ${ }^{11,13}$ and functionality of published PCP fusion proteins has not

54 been validated via rescue of orthologous mutant phenotypes. ${ }^{12,14-16}$ Furthermore, non-

55 physiological expression of exogenous proteins can mask subtle localization patterns or

56 signalling dynamics. This is highlighted by observations that in many occasions, co-expression

57 of another PCP component is required for asymmetric membrane localization of the fluorescent

58 fusion protein of interest. ${ }^{17-20}$

59 To understand the dynamic regulation of native PCP, we have aimed to overcome limitations

60 imposed by exogenous cell polarity reporters by imaging and manipulating the endogenous

61 zebrafish Vangl2 protein, a core PCP component and essential regulator of vertebrate planar

62 polarity. ${ }^{21,22}$ Using CRISPR/Cas9 gene editing, we introduced a superfolder GFP (sfGFP) tag

63 onto the N-terminus of endogenous Vang12 to generate a functional and native PCP reporter

64 protein. By performing detailed embryonic cell transplantation and single-cell level analyses, we

65 documented the dynamic sub-cellular localization of Vang12 over the course of neural tube 
66 formation, revealing novel associations of sfGFP-Vang12 positive cell membranes with polarized

67 basal bodies in floorplate cells. Furthermore, we exploited zGrad ${ }^{23}$ GFP-specific protein

68 degradation methodologies to conditionally interrogate sfGFP-Vangl2 function in floor plate

69 cells, demonstrating an essential role for Vangl2 in both the establishment and maintenance of

70 polarized basal body positioning as well as an inherent and surprising directionality to the

71 intercellular propagation of PCP. Our work highlights the importance and utility of studying

72 endogenous proteins in the context of PCP, and opens the door to conditional analysis of PCP

73 function beyond embryonic development.

\section{Results and Discussion}

76 To visualize the dynamic regulation of endogenous PCP signalling in zebrafish, we used

77 CRISPR/Cas9 genome editing to target a superfolder green fluorescent protein (sfGFP) onto the

78 N-terminus of Vangl2, an essential and membrane-localized PCP signalling protein (Fig. 1a;

79 targeting details in Methods section). Embryos carrying the knock-in allele showed prominent

80 sfGFP signal in the brain and spinal cord at 22 hours post fertilization (hpf) (Fig. 1b). In striking

81 contrast to the vangl2 ${ }^{m 209 / 209}$ loss-of-function mutant phenotype, which is embryonic lethal ${ }^{21}$, fish

82 homozygous for the $v a n g 2^{s f G F P}$ allele or trans-heterozygote for $v a n g l 2^{s f G F P}$ and $v a n g l 2^{m 209}$ alleles

83 are viable, fertile and appear morphologically normal (Fig. 1c). Closer inspection revealed that

84 vangl2 $2^{s f F P / S f G F P}$ embryos are slightly but significantly shorter than wildtype siblings at $24 \mathrm{hpf}$

85 (Fig. 1d). Interestingly, heterozygote vangl2 ${ }^{m 209 /+}$ embryos are similarly shorter than wildtype,

86 which has not been previously reported. Vangl2 $2^{\mathrm{s} G F P / m 209}$ transheterozygote embryos demonstrate

87 a further shortening of the body axis but are significantly less affected than the vangl2 $209 / m 209$

88 mutants (Fig. 1d). Altogether these data indicate that, although mildly hypomorphic, the sfGFP- 
89 Vang12 fusion protein is functional and can therefore be used to interrogate endogenous PCP $90 \quad$ signalling dynamics.

91 To determine the onset of Vangl2 expression, we imaged vangl2 $2^{\text {sfGFP/sfGFP }}$ embryos (hereafter

92 referred to as vangl2 ${ }^{s f G F P}$ ) through blastula and gastrula stages. Weak Vangl2 membrane

93 localization could be observed in a subset of cells as early as high stage ( $3.3 \mathrm{hpf}$ ), which became

94 more ubiquitous through early epiboly stages (4.6 hpf; Fig. 1e). Interestingly, weak Vangl2

95 membrane-localization was also observed within enveloping layer (EVL) cells at these stages

96 (Supplementary Fig. 1a). These observations are consistent with a role for Vang12 prior to

97 gastrulation, perhaps in promoting tissue cohesion in margin cells during early blastoderm

98 morphogenesis. ${ }^{24}$ Vang12 expression levels increased, and membrane localization became more

99 prominent with the onset of gastrulation at shield stage, persisting through $75 \%$ epiboly and bud

100 stages (Fig. 1e, Supplementary Fig. 1b-e). This corresponds to the known role for Vang12 in the

101 regulation of convergence and extension movements. ${ }^{21}$ Although immunohistochemical analyses

102 of Vang12 expression have yielded similar observations ${ }^{14}$ the superior consistency, sensitivity

103 and live-imaging capabilities of sfGFP-Vangl2 expression highlight its utility for experimental

104 studies.

105 In addition to membrane-localized Vang12, we also observed punctate intracellular

106 accumulations, which became prominent at the onset of gastrulation when they concentrated into

107 large cytosolic aggregates (Fig. 1e). To investigate the identity of these compartments, we 108 analysed Vang12 localization in relation to known markers of intracellular sorting organelles

109 (Fig. 1f-i and Supplementary Fig. 1b-e). In published studies, early and late endosome markers

110 Rab5 and Rab7 colocalize with PCP components Flamingo/Celsr and Frizzled-3a in Drosophila

111 wing, mouse keratinocytes and zebrafish neural tube. ${ }^{10,15,25-27}$ The recycling endosome marker 
112 Rab11 also colocalizes with Flamingo/Celsr in mouse skin and in Drosophila wing, and with

113 Vangl2 in cell culture. ${ }^{15,28,29}$ However, vertebrate studies have used exogenous PCP reporters,

114 which might not recapitulate the endogenous subcellular protein localization and may induce

115 trafficking artifacts. While we observed that Rab5c colocalized with some of the smaller Vang12

116 puncta at both shield and 90\% epiboly stages (Fig. 1f and Supplementary Fig. 1b), and that

117 Rab11a colocalized with Vang12 at the cell membrane but not within the cytoplasm (Fig. 1h and

118 Supplementary Fig. 1d), the majority of Vang12 puncta colocalized with mCherry-Rab7 (Fig. 1g

119 and Supplementary Fig. 1c) suggesting late endosomal transport to lysosomes or the trans-Golgi

120 network (TGN). Prior to gastrulation the zebrafish Golgi is fragmented, ${ }^{30}$ and whereas we could

121 see occasional sfGFP-Vang12 signal in the proximity of the TGN reporter dsRed-GalT, Vangl2

122 was predominantly localized elsewhere in the cell (Fig. 1i and Supplementary Fig. 1e). Taken

123 together these results suggest that, at early gastrulation stages, Vangl2 is targeted to late

124 endosomes for degradation.

125 Following gastrulation, strong Vang12 expression was observed in the central nervous system,

126 whereas only weak sfGFP-Vangl2 signal could be detected in surrounding axial and paraxial

127 mesodermal lineages (Figs. 1b, 2a). Previous work has demonstrated that exogenous GFP-

128 tagged Vang12 or its cytoplasmic binding partner Prickle polarize anteriorly in neuroepithelial

129 cells in zebrafish and Xenopus..$^{9,12,14,16,26,31}$ To characterize the asymmetric distribution of

130 endogenous Vang12, we performed cell transplantation at blastoderm stages to generate clones of

131 vangl2 ${ }^{s f G F P}$ cells within wildtype host embryos and imaged Vangl2 localization in individual

132 neuroepithelial cells at multiple time points during neural tube formation (Fig. 2b). At all stages,

133 weak sfGFP-Vang12 signal could be observed across the entire neuroepithelial cell membrane,

134 with bright membrane sfGFP-Vang12 domains enriched in puncta at anterior cell surfaces (Fig. 
135 2c, Supplementary Fig.2a, Supplementary Movie 1). Vangl2 localization varied considerably

136 between cells, including apical and basal distribution patterns, as well as localization into cell

137 protrusions (Fig. 2c and Supplementary Fig.2a). Previously, exogenous Vang12 reporter

138 localization has been quantified along single cellular planes. To gain a global view of Vang12

139 distribution, we performed 3-dimensional quantification of sfGFP-Vangl2 membrane-enrichment

140 in comparison to an RFP membrane reporter (mRFP) using the spot function in Imaris (detailed

141 description in Methods). We plotted the subset of membrane spots containing the highest sfGFP-

142 Vangl2 and mRFP fluorescent intensities across anterior-posterior and apico-basal cell axes

143 (Supplementary Fig.3A) and found that, despite the variation between cells, Vang12 was

144 significantly enriched at the anterior membrane at all stages analysed (Fig. 2d). Vangl2

145 localization was less uniformly polarized along the apico-basal axis, with Vangl2 showing

146 strongest apical enrichment at the 13 somite-stage concomitant to neural tube midline

147 formation $^{16}$ (Fig. 2e).

148 To examine the dynamics of Vang12 protein localization, we performed time-lapse imaging of

149 vangl2 $2^{\text {sfGFP }}$ chimeric embryos between 10-13 somite stages. Persistent sfGFP-Vangl2 enrichment

150 was observed along anterior membrane domains (Supplementary Movie 1), supporting evidence

151 from multiple Drosophila, Xenopus and mouse studies (predominantly using exogenous

152 reporters) that PCP components concentrate into potential "signalosomes" on the cell

153 membrane..$^{9,16,20,28,32-35}$ In Xenopus, exogenous Vang12 and Prickle reporters accumulate with

154 actomyosin at shrinking neuroepithelial cell junctions ${ }^{9}$ and, in mesodermal cells undergoing

155 convergence and extension movements, Prickle2 colocalizes with the LifeAct filamentous actin

156 reporter. ${ }^{35,36}$ These observations suggest that Vang12/PCP directly influences cytoskeletal re-

157 organization. To investigate the relationship between anterior Vang12 enrichment and actin 
158 organization, we co-expressed a mCherry-LifeAct reporter in vangl2 ${ }^{\text {sfGFP }}$ cells. Live imaging 159 revealed that the majority of neuroepithelial cell protrusive activity and LifeAct localization was 160 restricted to apical and basal surfaces, and not to anterior membranes where sfGFP-Vangl2 was 161 enriched (Supplementary Movies 2-3). However, sfGFP-Vangl2 could be observed accumulating 162 within active cellular protrusions at the anterior membrane (Supplementary Fig.2a,

163 Supplementary Movie 4), consistent with known roles for PCP and Vang12 in regulating 164 filopodia and cell protrusive activity during gastrulation and facial branchiomotor neuron 165 migration. ${ }^{11,12,37}$ Together, although the presence of bright sfGFP-Vang12 puncta supports the 166 notion that PCP signalling functions in specific subdomains on the anterior membrane, the 167 presence of Vangl2 membrane enrichment was not predictive of a particular membrane 168 behaviour.

169 To further understand how neighbouring cells affect asymmetric Vangl2 localization into 170 different membrane-domains, we performed cell transplantation experiments to generate clones 171 of vangl2 $2^{s f G P}$ cells within a vangl2 ${ }^{t k 50 f}$ loss-of-function host embryo (Fig. 2f). In this PCP172 deficient environment, vangl2 ${ }^{s f G F P}$ cells clustered together, suggesting that Vang12 expressing 173 cells are able to recognize and preferentially interact with one other. While Vangl2 remained 174 localized to the cell membrane, planar polarized membrane enrichment was lost and Vangl2 175 appeared to concentrate at cell protrusions (Fig. 2i and Supplementary Fig.2b). We picked a 176 subset of the most mediolaterally aligned cells to quantify asymmetric Vang12 localization and 177 found no difference in the distribution of the brightest Vangl2 and mRFP spots along the 178 anterior-posterior or apico-basal axes (Fig. 2g-h; Supplementary Fig. 3d). Loss of polarity in 179 vangl2 $2^{\text {sfGFP }}$ positive clones supports a cell non-autonomous regulation of vertebrate PCP. The 
180 persistence of Vang12 in cell protrusions suggests that it might function in mediating local

181 signalling that is independent of its role in global anterior-posterior neural tube planar polarity.

182 The re-organization and re-establishment of cell polarity over the course of cell division remains 183 a poorly understood feature of vertebrate PCP. In zebrafish, asymmetric localization of

184 exogenous Prickle-GFP reporter molecules is lost upon neuroepithelial cell division, but is re-

185 established as daughter cells reintegrate into the neuroepithelium. ${ }^{16}$ In mouse epidermis, different

186 studies have shown PCP components to be internalized during mitosis or to persist on cell

187 membranes in a polarized manner. ${ }^{15,38}$ Exogenous Vang12, specifically, has been shown to

188 persist on cell membranes and to get trans-internalized from neighbouring cell membranes into

189 the dividing cell. ${ }^{10}$ To determine the fate of endogenous Vang12 during mitosis, we imaged

190 vangl2 $2^{s f G P}$ neuroepithelial cells over the course of cell division. Notably, asymmetric sfGFP-

191 Vangl2 distribution was lost upon cell rounding, but Vang12 remained at the cell membrane

192 (Supplementary Movie 3-4). We observed sfGFP-Vang12 enriched at the poles of dividing cells,

193 as well as to basolateral membrane projections that are maintained during division

194 (Supplementary Movies 3-4). Although some intracellular sfGFP-Vangl2 signal was observed in

195 dividing cells, we did not observe significant endocytosis of membrane Vangl2 or trans-

196 endocytosis between neighbouring cells (Supplementary Movies 3-4). Reacquisition of Vangl2

197 polarization in daughter cells was not obvious immediately upon division (Supplementary Movie

198 2). Of interest, sfGFP-Vangl2 positive membrane protrusions from distant neighbours could be

199 observed interacting with dividing cells (Supplementary Movie 4). Out of 24 cells that had an

200 anterior Vangl2 positive protrusion, 11 were touching a dividing cell, and the same was observed

201 for $15 / 32$ apical protrusions (total $n=112$ cells). Our results show that Vangl2 remains on the 
membrane but loses its asymmetry during mitosis, and that re-establishment of cell polarity may be regulated by contacts from neighbouring cells.

204 In addition to cytoskeletal organization, polarized basal body (BB) positioning has been 205 identified as a conserved readout of PCP from insects to vertebrates. ${ }^{39}$ In zebrafish floorplate 206 cells, BBs are localized to the posterior apical membrane by $24 \mathrm{hpf}$, and the establishment of BB 207 polarity requires Vang12..$^{13,26,40}$ To examine the relationship between Vangl2 localization and BB 208 positioning, we imaged the floorplate of vangl $2^{s f G F P}$ embryos at 22 and $32 \mathrm{hpf}$. In the sagittal 209 plane, sfGFP-Vang12 was enriched on the anterior apical cell membrane (Fig. 3a-b), in 210 accordance with observations using an exogenous GFP-Vang12 transgenic reporter. ${ }^{12,26}$

211 However, in some floorplate cells apically enriched Vang12 appeared to extend anteriorly 212 towards neighbouring cells (Fig. 2a, Fig. 3a-b). This bright apical Vang12 signal was closely 213 associated with BBs labelled with Centrin-mCherry, and with Arl13b-positive cilia (Fig. 3a-b).

214 We imaged individual transplanted vangl2 $2^{s f G F P}$ floorplate cells and found that Vangl2 was indeed 215 localized asymmetrically on anterior cell membranes, and that in some cells it further localized 216 to an anterior apical projection (Fig. 3c). Labelling BBs in transplanted vangl2 ${ }^{s f G F P}$ cells with 217 Centrin-mCherry confirmed the proximity of anterior Vangl2 with posteriorly polarized BBs 218 (Fig. 3d). Furthermore, labelling of host cell BBs confirmed that Vangl2-positive apical 219 membrane closely associated with the posterior BB of neighbouring cells (Fig. 3e, 11/17 cells). 220 Of interest exogenous Frizzled3a, a putative intracellular binding partner of Vangl2, has been 221 shown to be enriched posteriorly into puncta at the base of cilia in zebrafish. ${ }^{26}$ This localization 222 pattern suggests that PCP activity could act to physically deform the floorplate cell membranes at 223 the site of BB docking, perhaps influencing the posterior tilting of the floorplate motile cilia. ${ }^{13}$ 
224 To determine whether Vang12 also controls the maintenance of BB polarity, we took advantage

225 of zGrad GFP-nanobody protein degradation methodologies to conditionally manipulate

226 endogenous sfGFP-Vangl2 protein levels. ${ }^{23} \mathrm{We}$ first confirmed that zGrad is non-toxic to

227 embryos, and that $z G r a d$-injected vangl2 $2^{s f F P / s f G F P}$ embryos phenocopy vangl2 loss-of-function

228 mutants (Supplementary Fig. 4a-b). Furthermore, because vangl2 mutants are subject to maternal

229 effects ${ }^{16}$, we investigated the consequences of $z$ Grad expression across a full genotypic spectrum

230 of maternal and zygotic vangl $2^{\text {sfGFP }}$ alleles. Strikingly, sequential zGrad-mediated degradation of

231 maternal and zygotic vangl $2^{s f G F P}$ gene products yielded progressively more severe phenotypes as

232 measured by embryo length and axial length-width ratios (Fig. 4a, Supplementary Fig. 4c),

233 recapitulating maternal and zygotic vangl2 mutant phenotypes. This highlights the sensitivity and

234 functionality of sfGFP-Vang12 zGrad protein degradation methods.

235 To conditionally manipulate sfGFP-Vangl2 protein levels within the floorplate, we generated a

236 ubiquitously expressed $\operatorname{Tg}(\beta$-actin2::loxP-mCherry-STOP-loxP-zGrad $)$ transgene and crossed it

237 to the $\operatorname{Tg}\left(\right.$ foxj 1 a::iCre) line $^{41}$, which expresses Cre recombinase (activating zGrad) specifically

238 within motile-ciliated cell lineages. At 32 hpf, when BB polarity has been established, sfGFP-

239 Vangl2 was still present at the membrane of experimental zGrad; foxjla::iCre; vangl2 ${ }^{\text {sfGFP }}$

240 floorplate cells (data not shown). However, mosaic loss of sfGFP-Vangl2 could be observed by

$24148 \mathrm{hpf}$ (Fig. 4b), allowing us to analyse both cell-autonomous and non-autonomous roles for

242 Vang12 in the maintenance of BB polarity. Images were scored for the loss of Vang12 in

243 individual cells and their immediate anterior and posterior neighbours. Irrespective of their

244 Vangl2 expression status, cells surrounded by sfGFP-negative neighbours demonstrated a loss of

245 BB polarity when compared to control populations. Strikingly, the presence of sfGFP-Vang12 in

246 a posterior neighbouring cell was associated with restored BB polarity in Vangl2-negative cells, 
247 even if the posterior neighbour demonstrated abnormal BB positioning (Fig. 4c-d). In contrast,

248 the presence of sfGFP-Vangl2 in anterior neighbours did not restore polarity of Vangl2 negative

249 cells. These results demonstrate that Vang12 is required for the maintenance of BB polarity, and

250 that Vangl2 functions non-cell autonomously to propagate PCP in a posterior to anterior

251 direction.

252 Taken together, our work provides essential new insights into the establishment and maintenance

253 of vertebrate PCP. Live imaging of endogenous Vang12 has authenticated broad principles

254 predicted using exogenously introduced reporter molecules, including organization of PCP

255 across the embryonic anterior-posterior axis and concentration of core PCP proteins into

256 'signalosomes' at the cell membrane. It has also yielded novel findings that 1) demonstrate

257 dynamic redistribution of Vang12 within discrete subcellular membrane domains over the course

258 of gastrulation and neurulation, 2) suggest tissue-specific contexts for published tenets regarding

259 membrane recycling of PCP proteins during mitosis or their association with the actin

260 cytoskeleton, and 3) indicate an inherent and surprising directionality to the intercellular

261 propagation of PCP. Outside of C. elegans, ${ }^{42}$ functional fluorescently-tagged PCP knock-in

262 alleles have not been widely reported. Our vangl2 $2^{s f G P}$ allele thus represents an invaluable

263 resource that, when combined with embryonic cell-transplantation, live-imaging and conditional

264 zGrad degradation methodologies, establishes a powerful experimental paradigm for future

265 investigations into vertebrate cell polarity. 
272 Animal husbandry

273 Zebrafish husbandry and experimental protocols were approved by the Hospital for Sick

274 Children's Animal Care Committee, and all protocols were performed in accordance with

275 Canadian Council on Animal Care guidelines. Wild-type zebrafish from TU strains were used.

276 The vangl2 mutant allele $t r i^{t k 50 f}$ was used for cell transplantation experiments ${ }^{21}$, and the $t r i^{m 209}$

277 allele was used in to validate the vangl2 ${ }^{s f G F P}$ allele functionality because of the ability to use a

278 published PCR mediated genotyping strategy ${ }^{21,43} \cdot \operatorname{Tg}($ foxjla::iCre) line has been previously

279 described ${ }^{41}$. Embryos from natural matings were grown at $28^{\circ} \mathrm{C}$ unless otherwise indicated. 280

281 sfGFP-Vangl2 targeting strategy

282 Target Selection

283 A previously validated guide RNA (gRNA) was used to target the sequence surrounding the start 284 codon of vangl2 (targeting sequence GGATAACGAGTCGCAGTACTCGG) ${ }^{44}$. As a proof of 285 principle, a solution containing $300 \mathrm{mM} \mathrm{KCl,} 100 \mathrm{ng}$ vangl2 gRNA, $200 \mathrm{ng}$ recombinant Cas9 286 (PNA Bio cat. CP01) and 10\% phenol red was incubated at $37^{\circ} \mathrm{C}$ for 5 minutes to form 287 ribonucleoprotein complexes (RNPs), and injected into wild-type embryos at the 1-cell stage. 288 Embryos were (i) scored for vangl2/trilobite phenotypes at $28 \mathrm{hpf}$ and (ii) assessed for loss of a 289 ScaI restriction site by PCR to gauge mutagenesis efficiency. 


\section{Targeting Vector Construction}

294 Prior to vector construction, male and female Tu wild-type animals were screened by PCR for 295 polymorphisms surrounding vangl2 exon 2 . Left and right arms of homology of $\sim 1-1.2 \mathrm{~kb}$ in 296 length were amplified from fin clips and subjected for Sanger sequencing. Fish containing highly

297 similar amplicons were selected for use in targeting experiments ( 4 males and 4 females). A 2.2

$298 \mathrm{~kb}$ amplicon surrounding vangl2 exon 2 was amplified from these wildtype fish and cloned into 299 pDONR221. A superfolder GFP (sfGFP) was amplified from sfGFP-N1 (a kind gift from

300 Michael Davidson and Geoffrey Waldo, Addgene \#54737) ${ }^{45}$ and cloned into the vangl2 targeting 301 intermediate with a 3x(GGGGS) flexible linker by megaprimer $\mathrm{PCR}^{46}$ and verified for in-frame 302 inclusion. Site-directed mutagenesis was performed on this intermediate to abolish the vangl2 303 gRNA targeting site to prevent vector from being cleaved in vivo. This final targeting 304 intermediate was amplified and cloned into XhoI and EcoRI sites of pKHR5 (a kind gift from D. 305 Grunwald, Addgene \#74593). All targeting intermediates and final clones were fully verified by 306 Sanger sequencing.

308 Knock-In Injections

309 A $2.5 \mu 1$ mix containing 20 ng of vangl2 targeting vector, $1.25 \mathrm{U}$ of I-SceI (NEB cat. R0694S), $3100.5 x$ Cutsmart buffer and 10\% phenol red was prepared. A second $2.5 \mu 1$ mix containing $300 \mathrm{mM}$ $311 \mathrm{KCl}, 100 \mathrm{ng}$ vangl2 gRNA and $200 \mathrm{ng}$ recombinant Cas9 (PNA Bio cat. CP01) was incubated at $31237^{\circ} \mathrm{C}$ for 5 minutes. The two mixes were combined together and one nanolitre was injected into 313 incrosses between pre-designated Tu wild-type animals. Mosaic GFP fluorescence was observed 314 in several F0 embryos which were separated and grown to adulthood. Adult F0 animals were 315 outcrossed to wildtype Tu fish and screened for GFP+ fluorescence. A founder transmitting 36\% 
316 of GFP+ embryos was identified, and F1 embryos were screened by PCR using the following

317 primers, F: CCGCGCTCTCCAGTCCGTCA, R: CGAGAGCTGCGTGAGTGTGAA, to ensure 318 precise editing of the vangl2 locus.

\section{Line Establishment and Maintenance}

321 The F1 fish were incrossed to produce homozygous F2 fish. Embryos were screened either 322 visually by GFP fluorescence or genotyped using the primers listed above.

\section{Transgenesis}

325 Transgenesis was performed using standard Gateway-compatible procedures ${ }^{47}$. The zGrad open 326 reading frame was amplified from pCS2(+)-zGrad (a kind gift from H. Knaut; Addgene 327 \#119716) and cloned into pDONR-P2rP3 to generate p3E-zGrad. To generate $\operatorname{Tg}(\beta a c t i n 2:: \operatorname{lox} P$ $328 m$ Cherry STOP loxP zGrad) zebrafish, p5E- $\beta a c t i n 2^{47}$, pME-loxP mCherry STOP loxP (a gift 329 from K. Kwan), and p3E-zGrad were recombined into pDEST Tol2 pA2 transgenesis vector. 330 Embryos were injected at the one-cell stage with 25 pg of assembled transgene and 25 pg of Tol2 331 mRNA. Embryos were sorted at $48 \mathrm{hpf}$ for mCherry reporter expression and were subsequently 332 grown to adulthood. Individuals were bred to TU wild-type zebrafish to generate stable F1 lines. 333 Clones were fully verified by Sanger sequencing.

\section{Plasmids and embryo microinjections}

336 Rab5c, 7 and 11a plasmids were a kind gift from B. Link, mCherry-LifeAct-7 was a kind gift 337 from M. Davidson (Addgene \#54491), pCS2(+)-zGrad was a gift from H. Knaut (Addgene \# 338 119716) ${ }^{23}$, pME-mTagBFP-CAAX was a gift from N. Cole (Addgene \# 75149) ${ }^{48}$, zebrafish 
339 codon-optimized mScarlet was a kind gift from T. Thiele, centrin-mCherry was a kind gift from

340 A. Salic and GalT-RFP was a kind gift from J. Wallingford. N-terminal mCherry-linker was

341 cloned into the Rab plasmids and mCherry-LifeAct and mTagBFP-CAAX were cloned into

342 pCS2+ using Gateway technology (Thermo Fisher Scientific). The plasmids were linearized and

343 sense-strand-capped mRNA was synthesized with the mMESSAGE mMACHINE system

344 (Ambion). 15 pg membrane-localized monomeric RFP (mRFP), 20 pg mTagBFP-CAAX, 30 pg

345 mCherry-LifeAct, 5-25 pg mCherry-Centrin, 2.5 pg Arl13b-mScarlet, 10 pg mCherry-Rab5c, 10

346 pg mCherry-Rab7, 15 pg mCherry-Rab11a, 10 pg GalT-RFP and 50-100 pg zGrad were injected

347 at one-cell stage.

\section{Cell transplantations}

350 Cell transplantations were performed at mid-blastula stages, as described previously ${ }^{49}$. For the 351 early somite stage imaging, embryos were grown at $25^{\circ} \mathrm{C}$ after transplantations.

\section{$353 \quad$ Imaging}

354 Brightfield embryonic imaging was performed on Leica M80 microscope with a MC170 HD

355 camera (Leica). Wide-field fluorescence imaging was performed on a Zeiss AXIO Zoom V16

356 microscope (EMS3/SyCoP3, Zeiss) and ORCA-Flash 4.0 C1140-22C camera (Hamamatsu).

357 Confocal imaging was performed on Leica TCS SP8 Lightning confocal with Leica DMI8

358 microscope, 40x/1.3 water immersion objective and HyD detectors using the 405, 488 and 552

359 laser lines (Leica). The images were deconvolved using the Lightning module. All time-lapse

360 imaging was performed using a resonant scanner with 16 times averaging. Maximum intensity

361 projections and time lapse images were recorded using Imaris (Bitplane). 


\section{Measurements of embryonic lengths and $\mathrm{L} / \mathrm{W}$ ratio}

363 Embryo lengths were measured by manually drawing a line from the front of the head to the tip

364 of the tail in ImageJ. Embryonic length/width ratios were calculated by measuring the widest and

365 longest points of krox 20 and myoD expression. Statistical testing was performed using GraphPad 366 Prism (GraphPad Software).

\section{Quantification of asymmetric Vangl2 membrane-localization}

369 Comparison of Vangl2 and mRFP membrane enrichment was performed using the spot function 370 in Imaris. In short, membrane-signals for mRFP or sfGFP were decorated with spots, and the 371 spots for the two channels were merged. A reference point was placed in the centre of each cell 372 to determine the anterior-posterior (X) and apical-basal (Y) axis. The position of each spot was 373 determined as their distance to the reference point. Mean fluorescence intensity for mRFP and 374 sfGFP within each spot was calculated, and the $10 \%$ of brightest spots for each fluorophore were 375 plotted against their normalized X and Y positions using GraphPad Prism (GraphPad Software). 376 Statistical testing was performed using GraphPad Prism (GraphPad Software).

\section{BB positioning quantification}

379 Basal body position was defined as a ratio between the distance of the basal body from the 380 posterior membrane and the anterior-posterior floor plate cell length normalized to 1.

381 Measurements were done in Imaris and statistical testing was performed using GraphPad Prism 382 (GraphPad Software). The values were divided into three categories: posterior (0.66-1), medial $383 \quad(0.33-0.66)$ and anterior $(0-0.33)$. 


\section{Whole-mount in situ hybridization}

386 Antisense RNA probes were prepared using DIG RNA labelling kit (Roche) from linearized

387 DNA. Whole mount RNA in situ hybridizations were performed according to Rose et al. ${ }^{50}$.

388 Samples were genotyped after imaging.

389

390

\section{References}

391

392 1. Devenport, D. Tissue morphodynamics: Translating planar polarity cues into polarized cell behaviors. Semin. Cell Dev. Biol. doi:10.1016/j.semcdb.2016.03.012. (2016)

394

2. Davey, C. F. \& Moens, C. B. Planar cell polarity in moving cells: think globally, act locally. Development 144, 187-200 (2017).

3. Humphries, A. C. \& Mlodzik, M. From instruction to output: Wnt/PCP signaling in development and cancer. Curr. Opin. Cell Biol. 51, 110-116 (2018).

4. Jussila, M. \& Ciruna, B. Zebrafish models of non-canonical Wnt/planar cell polarity signalling: fishing for valuable insight into vertebrate polarized cell behavior. Wiley Interdiscip. Rev. Dev. Biol. 6. (2017).

5. Butler, M. T. \& Wallingford, J. B. Planar cell polarity in development and disease. Nat. Rev. Mol. Cell Biol. 18, 375-388 (2017).

403 6. Carvajal-Gonzalez, J. M. \& Mlodzik, M. Mechanisms of planar cell polarity establishment in Drosophila. F1000Prime Rep. 6, 98 (2014). 
asymmetric morphogenesis of hair follicles. Nat. Cell Biol. 10, 1257-1268 (2008).

407 8. Montcouquiol, M. et al. Asymmetric localization of Vang12 and Fz3 indicate novel 408 mechanisms for planar cell polarity in mammals. J. Neurosci. 26, 5265-5275 (2006).

409 9. Butler, M. T. \& Wallingford, J. B. Spatial and temporal analysis of PCP protein dynamics during neural tube closure. eLife 7, e36456 (2018).

411 10. Heck, B. W. \& Devenport, D. Trans-endocytosis of planar cell polarity complexes during cell division. Curr. Biol. 27, 3725-3733.e4 (2017).

413 11. Love, A. M., Prince, D. J. \& Jessen, J. R. Vangl2-dependent regulation of membrane 414 protrusions and directed migration requires a fibronectin extracellular matrix.

$415 \quad$ Development. 145, dev165472 (2018).

416 12. Davey, C. F., Mathewson, A. W. \& Moens, C. B. PCP Signaling between migrating 417 neurons and their planar-polarized neuroepithelial environment controls filopodial 418 dynamics and directional migration. PLOS Genet. 12, e1005934 (2016).

419 13. Borovina, A., Superina, S., Voskas, D. \& Ciruna, B. Vangl2 directs the posterior tilting 420 and asymmetric localization of motile primary cilia. Nat. Cell Biol. 12, 407-412 (2010).

421 14. Roszko, I., S. Sepich, D., Jessen, J. R., Chandrasekhar, A. \& Solnica-Krezel, L. A 422 dynamic intracellular distribution of Vang12 accompanies cell polarization during zebrafish gastrulation. Development 142, 2508-2520 (2015).

424 15. Devenport, D., Oristian, D., Heller, E. \& Fuchs, E. Mitotic internalization of planar cell 425 polarity proteins preserves tissue polarity. Nat. Cell Biol. 13, 893-902 (2011).

426 16. Ciruna, B., Jenny, A., Lee, D., Mlodzik, M. \& Schier, A. F. Planar cell polarity signalling 
couples cell division and morphogenesis during neurulation. Nature 439, 220-224 (2006).

428 17. Panousopoulou, E., Tyson, R. A., Bretschneider, T. \& Green, J. B. A. The distribution of

429 Dishevelled in convergently extending mesoderm. Dev. Biol. 382, 496-503 (2013).

430 18. Chu, C.-W. \& Sokol, S. Y. Wnt proteins can direct planar cell polarity in vertebrate 431 ectoderm. eLife 5, e16463 (2016).

432 19. Witzel, S., Zimyanin, V., Carreira-Barbosa, F., Tada, M. \& Heisenberg, C. P. Wnt11 433 controls cell contact persistence by local accumulation of Frizzled 7 at the plasma membrane. J. Cell Biol. 175, 791-802 (2006).

20. Butler, M. T. \& Wallingford, J. B. Control of vertebrate core planar cell polarity protein localization and dynamics by Prickle 2. Development 142, 3429-39 (2015).

437 21. Jessen, J. R. et al. Zebrafish trilobite identifies new roles for Strabismus in gastrulation and neuronal movements. Nat. Cell Biol. 4, 610-615 (2002).

22. Park, M. \& Moon, R. T. The planar cell-polarity gene stbm regulates cell behaviour and cell fate in vertebrate embryos. Nat. Cell Biol. 4, 20-25 (2002).

23. Yamaguchi, N., Colak-Champollion, T. \& Knaut, H. ZGrad is a nanobody-based degron system that inactivates proteins in zebrafish. eLife 8, e43125 (2019).

443 24. Petridou, N. I., Grigolon, S., Salbreux, G., Hannezo, E. \& Heisenberg, C. P. Fluidization444 mediated tissue spreading by mitotic cell rounding and non-canonical Wnt signalling. Nat. Cell Biol. 21, 169-178 (2019).

446 25. Mottola, G., Classen, A.-K., González-Gaitán, M., Eaton, S. \& Zerial, M. A novel function for the Rab5 effector Rabenosyn-5 in planar cell polarity. Development 137, 
449

450

451

452

453

454

455

456

457

458

459

460

461

462

463

464

465

466

467

468

26. Mathewson, A. W., Berman, D. G. \& Moens, C. B. Microtubules are required for the maintenance of planar cell polarity in monociliated floorplate cells. Dev. Biol. 452, 21-33 (2019).

27. Strutt, H. \& Strutt, D. Differential stability of flamingo protein complexes underlies the establishment of planar polarity. Curr. Biol. 18, 1555-1564 (2008).

28. Strutt, H., Warrington, S. J. \& Strutt, D. Dynamics of core planar polarity protein turnover and stable assembly into discrete membrane subdomains. Dev. Cell 20, 511-525 (2011).

29. Williams, B. B. et al. VANGL2 regulates membrane trafficking of MMP14 to control cell polarity and migration. J. Cell Sci. 125, 2141-2147 (2012).

30. Sepich, D. S. \& Solnica-Krezel, L. Intracellular Golgi Complex organization reveals tissue specific polarity during zebrafish embryogenesis. Dev. Dyn. 245, 678-691 (2016).

31. Ossipova, O., Kim, K. \& Sokol, S. Y. Planar polarization of Vang12 in the vertebrate neural plate is controlled by Wnt and Myosin II signaling. Biol. Open 4, 722-730 (2015).

32. Strutt, H., Gamage, J. \& Strutt, D. Robust asymmetric localization of planar polarity proteins is associated with organization into signalosome-like domains of variable stoichiometry. Cell Rep. 17, 2660-2671 (2016).

33. Stahley, S. N., Basta, L. P., Sharan, R. \& Devenport, D. Celsr1 adhesive interactions mediate the asymmetric organization of planar polarity complexes. eLife 10, e62097 (2021).

34. Shi, D. et al. Dynamics of planar cell polarity protein Vangl2 in the mouse oviduct 
epithelium. Mech. Dev. 141, 78-89 (2016).

470 35. Shindo, A., Inoue, Y., Kinoshita, M. \& Wallingford, J. B. PCP-dependent transcellular

471 regulation of actomyosin oscillation facilitates convergent extension of vertebrate tissue.

472 Dev. Biol. 446, 159-167 (2019).

473 36. Riedl, J. et al. Lifeact: a versatile marker to visualize F-actin. Nat. Methods 5, 605-607

$474 \quad(2008)$

475 37. Brunt, L. et al. Vang12 promotes the formation of long cytonemes to enable distant Wnt/ $\beta$ -

476 catenin signaling. Nat. Commun. 12, 2058 (2021).

477 38. Oozeer, F., Yates, L. L., Dean, C. \& Formstone, C. J. A role for core planar polarity

39. Carvajal-Gonzalez, J. M., Roman, A.-C. \& Mlodzik, M. Positioning of centrioles is a 481 conserved readout of Frizzled planar cell polarity signalling. Nat. Commun. 7, 11135

482 (2016).

483

40. Donati, A., Anselme, I., Schneider-Maunoury, S. \& Vesque, C. Planar polarization of cilia in the zebrafish floor-plate involves Par3-mediated posterior localization of highly motile basal bodies. Development 148, dev196386 (2021).

486 41. Van Gennip, J. L. M., Boswell, C. W. \& Ciruna, B. Neuroinflammatory signals drive 487 spinal curve formation in zebrafish models of idiopathic scoliosis. Sci. Adv. 4, 1-12 (2018). 
tagging-based screen reveals localized players in Wnt-directed asymmetric cell division. Genetics 208, 1147-1164 (2018).

492

493

494

495

496

497

498

499

500

501

502

503

504

505

506

507

508

509

510

43. Gao, B. et al. Wnt signaling gradients establish planar cell polarity by inducing Vangl2 phosphorylation through Ror2. Dev. Cell 20, 163-176 (2011).

44. Shah, A. N., Davey, C. F., Whitebirch, A. C., Miller, A. C. \& Moens, C. B. Rapid reverse genetic screening using CRISPR in zebrafish. Zebrafish 13, 152-153 (2016).

45. Pédelacq, J.-D. et al. Engineering and characterization of a superfolder green fluorescent protein. Nat. Biotechnol. 24, 79-88 (2006).

46. Geiser, M., Cèbe, R., Drewello, D. \& Schmitz, R. Integration of PCR fragments at any specific site within cloning vectors without the use of restriction enzymes and DNA ligase. BioTechniques 31, 88-92 (2001).

47. Kwan, K. M. et al. The Tol2kit: A multisite gateway-based construction Kit for Tol2 transposon transgenesis constructs. Dev. Dyn. 236, 3088-3099 (2007).

48. Don, E. K. et al. A Tol2 Gateway-Compatible Toolbox for the study of the nervous system and neurodegenerative disease. Zebrafish 14, 69-72 (2017).

49. Ho, R. K. \& Kane, D. A. Cell-autonomous action of zebrafish spt-1 mutation in specific mesodermal precursors. Nature 348, 728-730 (1990).

50. Rose, C. D. et al. SCO-Spondin defects and neuroinflammation are conserved mechanisms driving spinal deformity across genetic models of idiopathic scoliosis. Curr. Biol. 30, 2363-2373.e6 (2020). 


\section{Acknowledgements}

512 We gratefully acknowledge the SickKids’ Imaging Facility for assistance with confocal

513 microscopy and the SickKids' Zebrafish Facility technicians for excellent zebrafish care. This

514 work was supported, in part, by funding from Canadian Institutes of Health Research (FDN-

515 167285) and the Canada Research Chair program to B.C., and the Sigrid Juselius Foundation to

516 M.J.

517

518 Author Contributions

519 M.J. and C.W.B. performed Vang12 targeting, M.J. performed all Vang12 knock-in line

520 validation and Vang12 imaging experiments and analysis, C.W.B. generated the zGrad transgene,

521 B.C. supervised research studies, M.J. and B.C. wrote the manuscript with input from C.W.B.

522

523 Declaration of Interests

524 The authors declare no competing interests.

525

526 Supplementary Movie Titles

527 Supplementary Movie 1. Vang12 enrichment on anterior membranes is dynamic over time

528 Supplementary Movie 2. Vang12 enrichment on the anterior membrane does not colocalize with

529 filamentous actin

530 Supplementary Movie 3. Vang12 localizes to anterior apical membranes and to basal membrane

531 extensions in dividing cells

532 Supplementary Movie 4. Vang12 anterior polarization is lost during cell division 
Figure 1. Jussila et al.

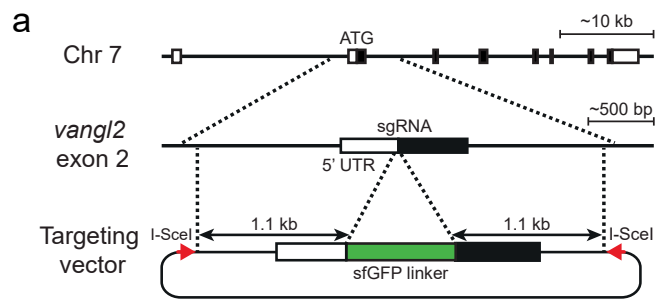

b

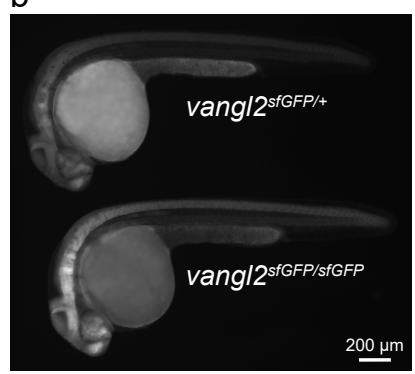

C

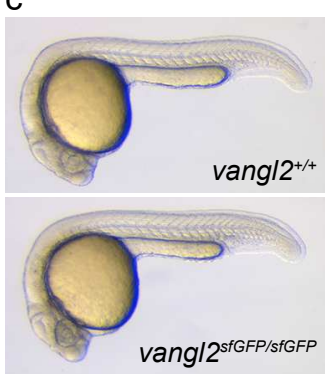

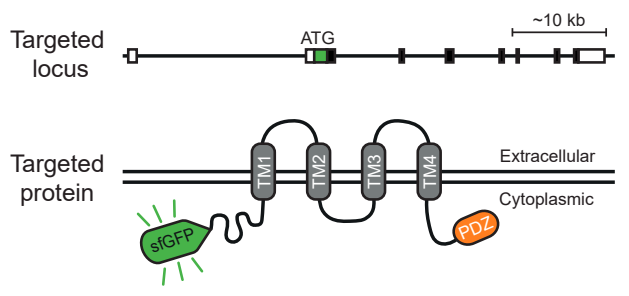

d

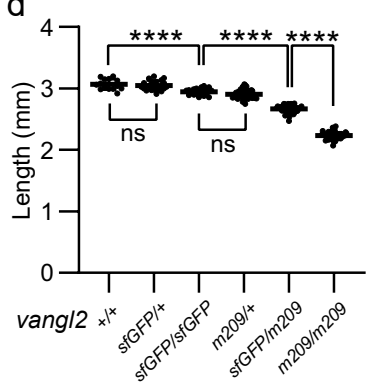

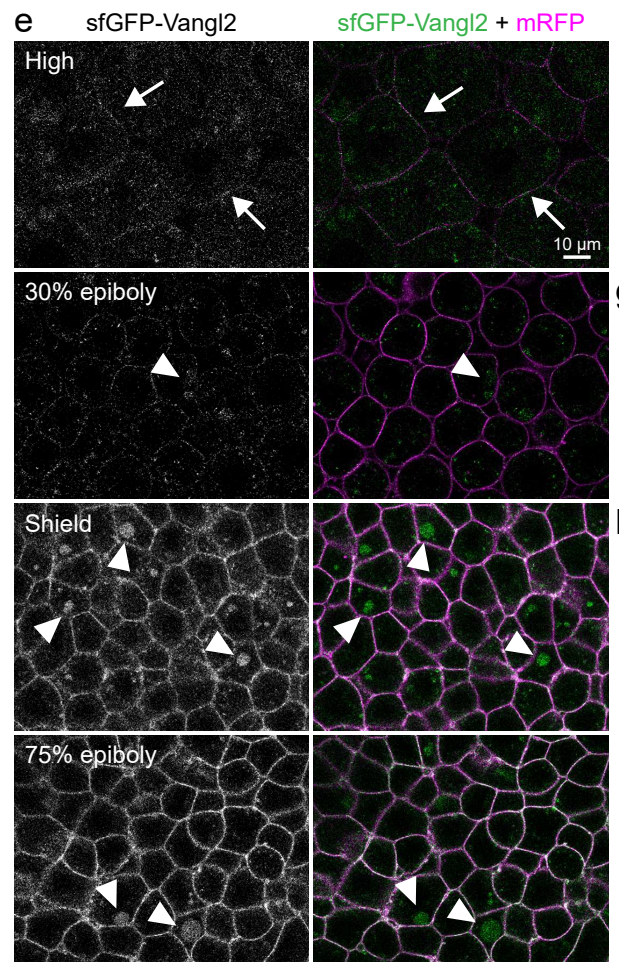
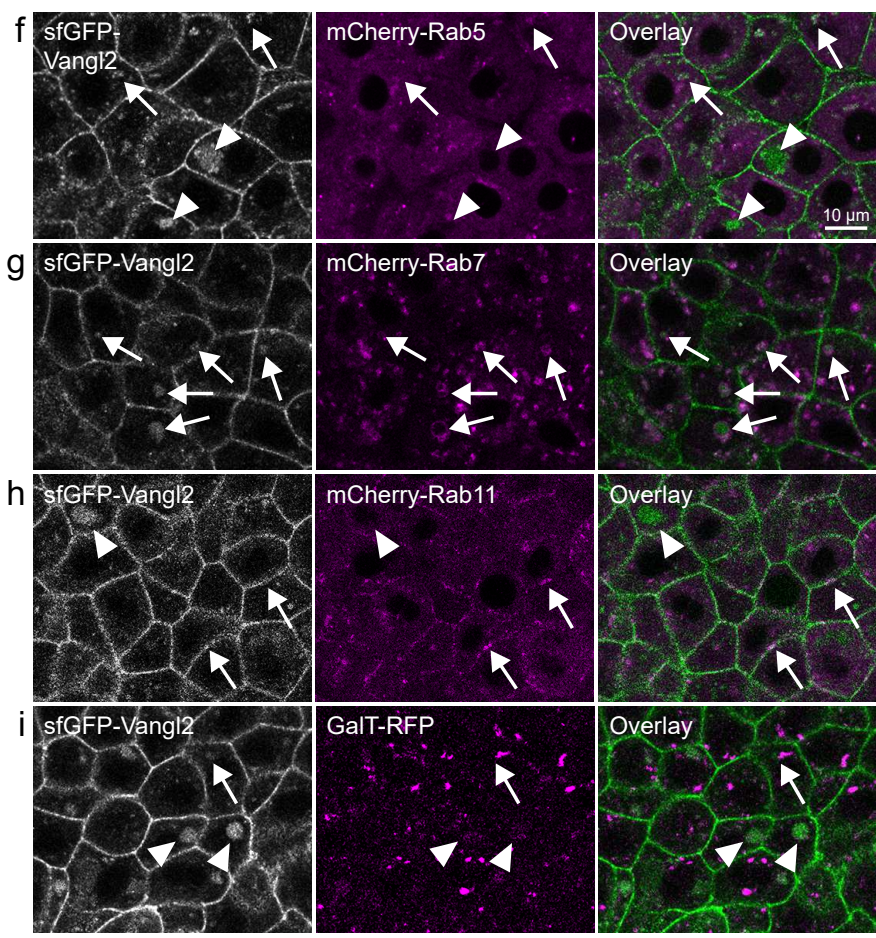

Figure 1. sfGFP-Vangl2 is functional and membrane localized during early zebrafish development (a) A schematic illustration of the sfGFP-Vangl2 knock-in targeting strategy.

(b) Wide-field fluorescence images demonstrating sfGFP-Vangl2 expression in $v a n g l 2^{s f G F P / 4}$ and $v a n g l 2^{s f G F P / s f G F P}$ embryos at 28 hours post fertilization (hpf).

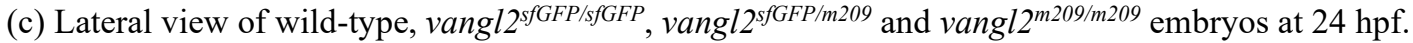

(d) Embryo length quantification at $24 \mathrm{hpf}$. Data shows representative experiments combined from independent crosses that were repeated 2-4 times. Statistical analysis was performed using one-way ANOVA with Tukey's

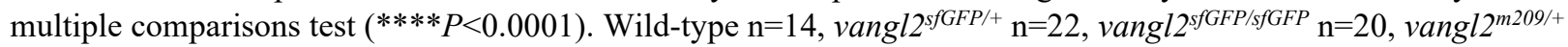
$\mathrm{n}=82$, vangl2 $2^{\text {sfGFP/m209 }} \mathrm{n}=72$, vangl $2^{m 209} \mathrm{n}=63$.

(e) Live confocal images of vangl2 $2^{s f G F P / s f G F P}$ (hereafter referred as vangl2 $2^{s f G F P}$ ) embryos at blastula through early gastrula stages, as indicated. Ectodermal cells were imaged in gastrulating embryos. Embryos were injected with mRNA coding for a membrane-localized monomeric RFP (mRFP) reporter. All sfGFP images were acquired using identical settings.

(f-i) Live confocal images of shield staged vangl $2^{\text {sfGFP }}$ embryos injected with mRNA coding for mCherry-Rab5c (f), mCherry-Rab7 (g), mCherry-Rab11a (h) or GalT-RFP (i) reporter constructs. Arrows point at cytoplasmicVangl2 puncta in close proximity to respective reporters, and arrowheads point at isolated Vangl2 puncta. 
Figure 2. Jussila et al.
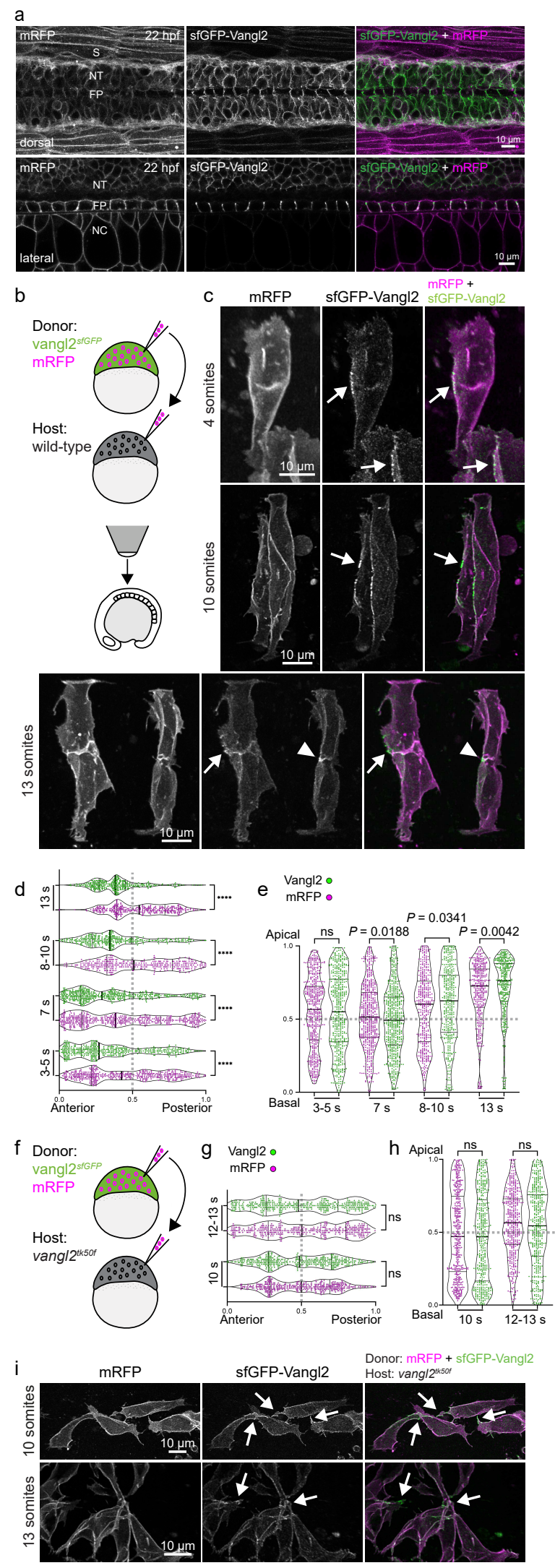
Figure 2. Planar polarized enrichment of Vangl2 on anterior neuroepithelial membranes is controlled by cell non-autonomous PCP signalling

(a) Confocal images comparing sfGFP-Vangl2 and mRFP localization at 22 hours post fertilization (hpf) in dorsal (top) and lateral (bottom) orientations. Anterior is to the left in all images. Strong Vangl2 expression is observed on cell membranes in the neural tube (NT) including the floor plate (FP), while only weak expression is observed in somites (S) and notochord (NC).

(b) A schematic illustrating transplantation of mRFP-labelled vangl2 ${ }^{\text {sfGPP }}$ donor cells into WT (wild-type) host embryos, at sphere stage. Chimeric embryos were imaged dorsally, at positions between the $2^{\text {nd }}$ and $8^{\text {th }}$ somite.

(c) Confocal images of mRFP and sfGFP-Vangl2 localization in the developing spinal cord of chimeric embryos at 4-somite, 10-somite and 13-somite stages of development. Maximum intensity projections are shown. Arrows point to Vangl2 enrichment on anterior neuroepithelial cell membranes, and arrowheads to anterior apical membranes at the neural midline.

(d-e) Distribution of the brightest sfGFP-Vang12 and membrane-RFP spots along anterior-posterior (d) and apicalbasal (e) axes of vangl2 $2^{s f G P}$ neuroepithelial cells, quantified at four consecutive stages of neural tube morphogenesis. Data from multiple transplanted vangl $2^{\text {sfGFP }}$ cells was pooled (3-5 somites, $\mathrm{n}=7 ; 7$ somites, $\mathrm{n}=7$; 8 -10 somites, $\mathrm{n}=6 ; 13$ somites, $\mathrm{n}=5$ ) and axial lengths normalized to 1 . Details of the quantification can be found in the Methods section. Statistical analysis was performed using a two-tailed Mann-Whitney test.

(f) A schematic illustrating transplantation of mRFP-labelled vangl2 sfGFP donor cells into vangl2 ${ }^{\text {tk5of }}$ loss-of-function host embryos, at sphere stage.

(g-h) Distribution of the brightest sfGFP-Vang12 and membrane-RFP spots along anterior-posterior (g) and apicalbasal (h) axes of vangl2 $2^{s f G F P}$ neuroepithelial cells transplanted into a vangl2 ${ }^{t k 50 f}$ mutant host, quantified at two consecutive stages of neural tube morphogenesis. Data from multiple transplanted vangl2sfGFP cells was pooled (10 somites, $\mathrm{n}=7$; $12-13$ somites, $\mathrm{n}=9$ ).

(i) Confocal images of mRFP and sfGFP-Vangl2 localization in vangl2 ${ }^{s f G F P}$ neuroepithelial cells within vangl2 ${ }^{\text {tk50f }}$ mutant hosts, at 10-somite and 13-somite stages of development. Maximum intensity projections are shown. Arrows point at Vangl2 enrichment on cell protrusions. 
Figure 3. Jussila et al.

a

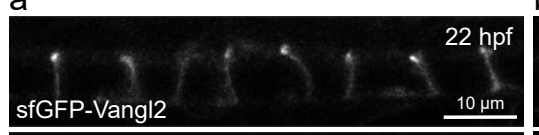

b
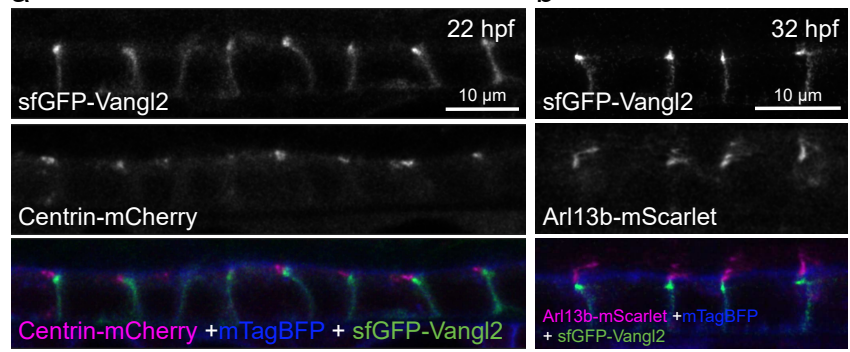

C

Donor: sfGFP-Vangl2 + mRFP

Host: unlabelled
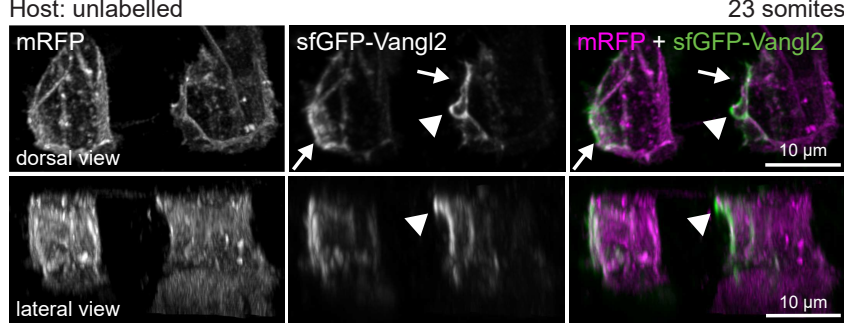

e

d

Donor: sfGFP-Vangl2

Donor: sfGFP-Vangl2 + Centrin-mCherry

Host: unlabelled

Host: mTagBFP

+ Centrin-mCherry

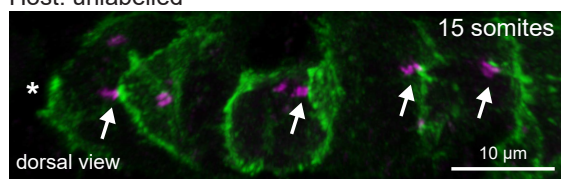

2. 15 somites
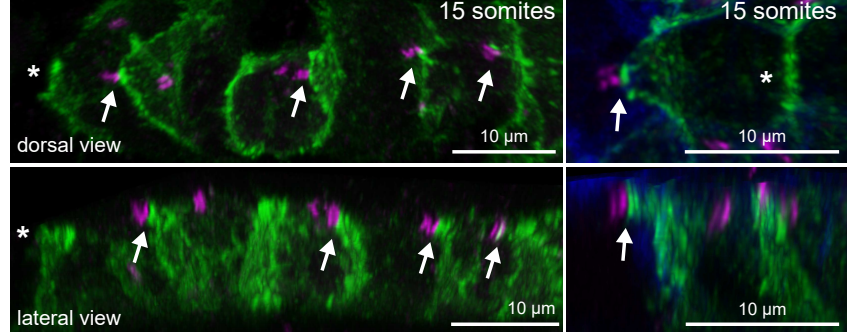

Figure 3. Vangl2-positive anterior membrane closely associates with planar polarized basal bodies of floor plate cells

(a) Confocal images of sfGFP-Vang12 localization in relation to basal body (Centrin-mCherry) and membrane (mTagBFP) reporters within floorplate cells of a vangl2 ${ }^{\text {sfGPP }}$ embryo at $22 \mathrm{hpf}$. Lateral view, anterior to the left.

(b) Confocal images of sfGFP-Vangl2 localization in relation to cilia axoneme (Arl13b-mScarlet) and membrane (mTagBFP) reporters within floorplate cells of a vangl2 ${ }^{\text {sfGPP }}$ embryo at $32 \mathrm{hpf}$. Lateral view, anterior to the left.

(c) Confocal image comparing sfGFP-Vangl2 and mRFP localization in floorplate cells of a 23-somite staged chimeric embryo ( $m R F P$ mRNA-injected vangl2sfGFP cells transplanted into WT hosts). Maximum intensity projections, dorsal (top) and lateral (bottom) views are shown, anterior to the left. Arrows point at Vangl2 enrichment on anterior cell membranes. Arrowhead points at Vangl2 enriched membrane protruding from the anterior apical surface.

(d) Confocal image comparing sfGFP-Vangl2 and Centrin-mCherry localization in floorplate cells of a 15-somite staged chimeric embryo (Centrin-mCherry mRNA-injected vangl2 ${ }^{s f G F P}$ cells transplanted into WT hosts). Maximum intensity projections, dorsal (top) and lateral (bottom) views are shown, anterior to the left. Arrows point at Vangl2 on the anterior membrane that is closely associated with a basal body docked on the posterior membrane. Asterisk indicates anterior unlabelled host cell that is not Centrin-mCherry positive.

(e) Confocal image comparing sfGFP-Vangl2 localization in relation to basal body (Centrin-mCherry) and membrane (mTagBFP) reporters in neighbouring floorplate cells of a 15-somite staged chimeric embryo (vangl2 ${ }^{s f G F P}$ cells transplanted into WT hosts injected with Centrin-mCherry and mTagBFP mRNA). Maximum intensity projections, dorsal (top) and lateral (bottom) views are shown, anterior to the left. Arrow points at Vangl2 on the anterior membrane of a donor cell that is closely associated with a basal body docked on the posterior membrane of a host cell. 
Figure 4. Jussila et al.
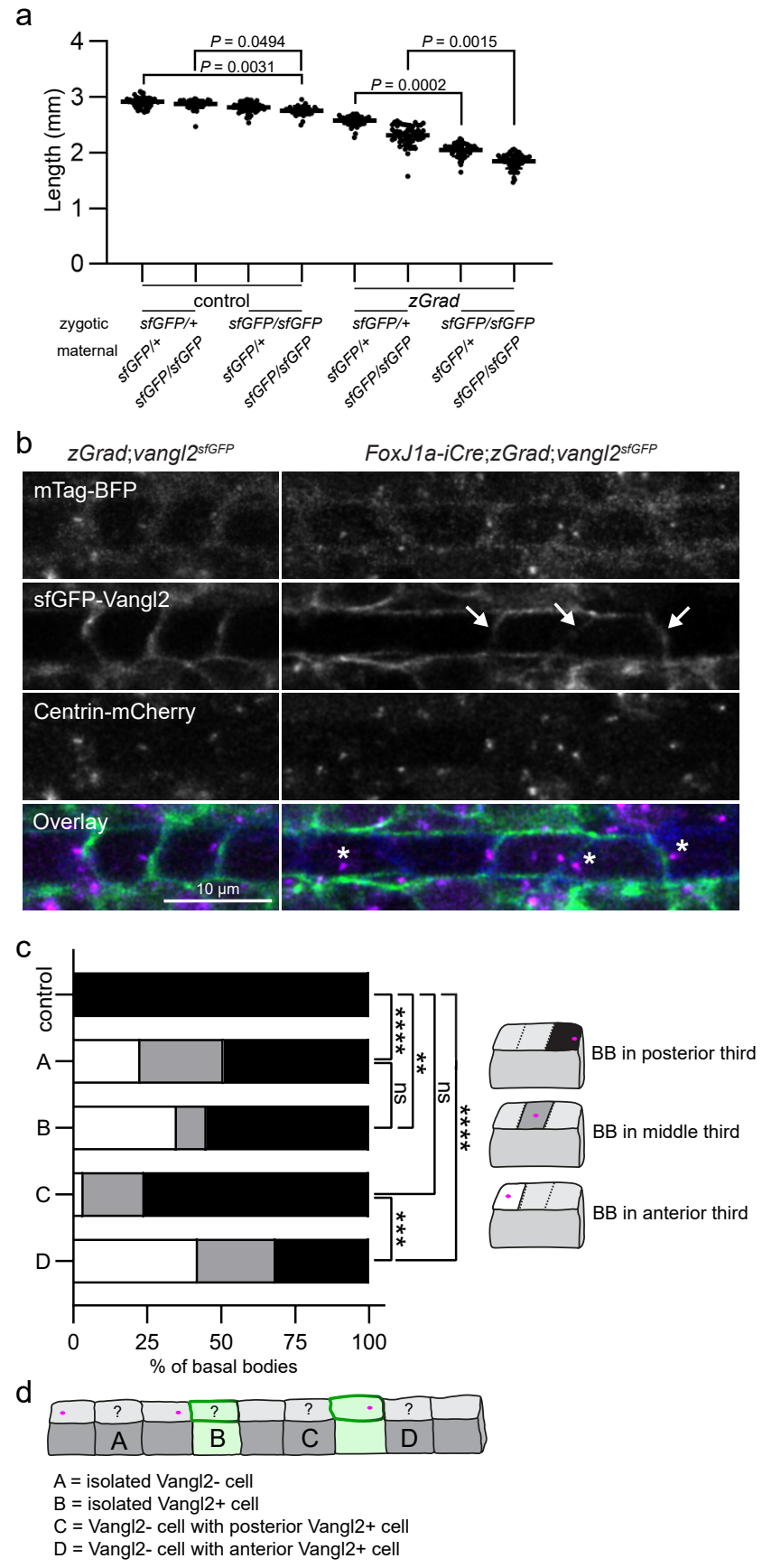

Figure 4. Conditional degradation of sfGFP-Vangl2 reveals a cell non-autonomous requirement for Vangl2 in maintaining basal body polarity

(a) Quantification of embryo lengths for zGrad mRNA-injected and control embryos at $24 \mathrm{hpf}$. Maternal and zygotic genotypes are as indicated. Data is pooled from three independent experiments. Control $\mathrm{n}$ from left to right: $\mathrm{n}=53$, $\mathrm{n}=62, \mathrm{n}=61, \mathrm{n}=36$. $\mathrm{zGrad} \mathrm{n}$ from left to right: $\mathrm{n}=55, \mathrm{n}=51, \mathrm{n}=53, \mathrm{n}=54$. Statistical analysis was performed using Kruskal-Wallis test with Dunn's multiple comparisons test.

(b) Confocal images of sfGFP-Vangl2 expression in relation to basal body (Centrin-mCherry) and membrane (mTagBFP) reporters within floorplate cells of control vangl2 ${ }^{s f G F P}$; $\operatorname{Tg}(\operatorname{lox} P$-mCherry-STOP-loxP-zGrad) embryos (left) and experimental vangl2 ${ }^{\text {sfFFP }}$; $T$ (loxP-mCherry-STOP-loxP-zGrad); Tg(foxjla-iCre) embryos (right) at 48 hpf. Dorsal view, anterior to the left. Arrows indicate residual sfGFP-Vangl2 expression in a subset of floorplate cells following zGrad expression. Asterisks indicate mispolarized basal bodies following zGrad expression.

(c) Quantification of basal body localization within the posterior, medial or anterior thirds of floor plate cells for control and vangl2 ${ }^{\text {sfGFP }}$; Tg(loxP-mCherry-STOP-loxP-zGrad); Tg(foxjla-cre) embryos described in (b). Cells were categorized as A-D based on the presence or absence of sfGFP-Vangl2 signal within both the cell and its immediate neighbours, as illustrated in (d). Control $n=164, A$ n=256, B n=20, C n=29, D n=19.

(d) A schematic illustrating cell categories quantified in (c). Grey colour indicates cells that have lost sfGFP-Vangl2 signal; green colour indicates residual sfGFP-Vangl2 expression. 


\section{Supplementary Files}

This is a list of supplementary files associated with this preprint. Click to download.

- JussilaetalMovie3NatComm.mp4

- JussilaetalMovie2NatComm.mp4

- JussilaetalSupplementaryFiguresNatComm.pdf

- JussilaetalMovie1NatComm.mp4

- JussilaetalMovie4NatComm.mp4

- JussilaetalSupplementaryFiguresNatComm.pdf

- nrreportingsummaryNCOMMS2129897.pdf 